\title{
PLATAFORMA DE GESTIÓN EDUCATIVA DE DISEÑO PROPIO
}

\author{
EDUCATIONAL MANAGEMENT PLATFORM \\ OF OUR OWN DESIGN
}

\begin{abstract}
ב?
Isidro José Pacheco Carrión ${ }^{a^{*}}$ y María Yolanda Jiménez Rubio ${ }^{a}$
\end{abstract}

Fechas de recepción y aceptación: 16 de julio de 2019, 4 de diciembre de 2019

Resumen: Actualmente, existe en el mercado un gran número de plataformas informáticas que permiten automatizar los diferentes procesos relacionados con la gestión de una institución educativa: pasar lista, horarios de los profesores, introducir notas, generación de calendarios comunes, envío de correos entre los distintos estamentos, comunicaciones con las familias, etc. Desde los colegios, se invierte una gran cantidad de esfuerzos, tanto económicos como personales, para que dichas aplicaciones sean adoptadas de forma masiva por todos los componentes de la institución, ya que, obviamente, una vez seleccionada una solución u otra, se deben introducir los datos (nombres de alumnos, de profesores, encargados de dirección, etc.) y se debe, además, formar a los profesores en el uso de la herramienta, con la consiguiente adaptación tanto de su metodología didáctica, como de su forma de trabajar. En el presente documento, se describe el proceso seguido para la creación e implantación de una plataforma propia de gestión de un colegio, creada a partir de las necesidades observadas en el día a día de los docentes.

Palabras clave: gestión educacional, educación, tecnología educacional, tecnología de la información, comunicación.

Abstract: Nowadays, there are a large number of web platforms at the market to make the different processes related to the management of an educational institution automatic:

${ }^{\text {a }}$ Facultad de Educación. Universidad Internacional de Valencia - VIU

* Correspondencia: Universidad Internacional de Valencia - VIU. Facultad de Educación. Calle Pintor Sorolla, 21. 46002 Valencia. España.

E-mail: isidrojose.pacheco@campusviu.es 
attendance register, teachers' schedules, introduction of grades, generating common calendars, mailings between the different levels, communication with families, etc. From schools, a lot of effort, both economic and personal, is invested for such applications to be adopted en masse by all members of the institution, since, obviously, once we choose a solution, we have to introduce new data (names of students, teachers, managers addresses, etc.) and to train teachers in the use of the tool selected, with the resulting adjustment of both his/her teaching methodology, and his/her way of working with students. In this document, the process for the creation and implementation of a management platform is described, as created from the needs observed in the daily life of teachers.

Keywords: educational management, education, education technology, information technology, comunication.

\section{INTRODUCCIÓN}

"Las nuevas tecnologías de la información y la comunicación (TICs) se han convertido en una herramienta insustituible y de indiscutible valor y efectividad en el manejo de la información con propósitos didácticos" (Canós y Canós, 2009). Ahora bien, de la misma manera, estas herramientas deben facilitar también la gestión docente, como, por ejemplo, pasar la lista de clase, poner notas a los alumnos, gestionar tutorías, etc., sobre todo desde el punto de vista de una institución educativa no universitaria, en donde todos estos datos deben estar actualizados prácticamente en tiempo real y en el que múltiples profesores ejercen su función docente en un mismo grupo, con la complejidad que esto añade, pues todos generan información sobre el alumno que debe unificarse de forma eficaz y a la mayor brevedad posible.

En los últimos años son muchos los centros educativos que han incorporado un sistema informático que les ayude con la gestión educativa, financiera, administrativa y de comunicación entre las personas adscritas a dichas instituciones. Sin embargo, este tipo de sistemas no se usa de forma generalizada en todos los centros por diferentes motivos: económicos, complejidad para adaptarse, etc. (Muñoz y Santini, 2015).

Hoy en día existen multitud de herramientas de gestión educativa en el mercado, a las que los centros pueden optar, como son, por ejemplo, las siguientes (Educación 3.0, 2018): 
- Alexia: Plataforma de gestión y comunicación presente en más de 1.200 centros educativos que permite optimizar la gestión académica y económica y la comunicación con la comunidad educativa (Alexia, 2019).

- Educamos: Permite la gestión eficiente de todos los procesos que tienen lugar en el día a día de la escuela, la comunicación abierta entre profesores, alumnos y familias que cohesionan la comunidad educativa y el aprendizaje innovador y personalizado gracias a la utilización de múltiples contenidos, soportes y procesos didácticos. "En España, más de 1.200 colegios, 50.000 profesores y 860.000 alumnos de todas las Comunidades Autónomas ya son usuarios" (SM, 2019).

- Additio App: Disponible desde la web y también para iOS y Android. Es un cuaderno de notas utilizado para que los docentes planifiquen su curso; permite controlar la asistencia y realizar un seguimiento de las notas de los alumnos. Dentro de sus características destacan: creación de tutorías de aviso, personalización de horarios, visualizar de forma semanal las tareas correspondientes, evaluaciones... Permite trabajar sin conexión a internet sincronizando los datos entre dispositivos. Posibilita organizar mejor las clases, trabajar de forma colaborativa y fomentar el aprendizaje de los estudiantes a la vez que se impulsa la participación de las familias (Additio App, 2019).

- Clickedu: Es una plataforma en la nube para facilitar a todos los estamentos del centro sus tareas habituales. Incluye la gestión económica, la comunicación con las familias, la gestión administrativa, la calidad y un entorno virtual de aprendizaje con un sistema de evaluación flexible, trabajo por proyectos y rúbricas. Dispone de una app para poder utilizar la plataforma a través de tabletas y smartphones con sistemas operativos iOS y Android (Clickedu, 2019).

El acceso a estas plataformas supone un desembolso económico para los colegios, además de la adaptación metodológica que deben hacer los profesores para acomodarse de forma satisfactoria a la solución cerrada que ofrecen. En este documento se presenta una plataforma de gestión educativa, creada a partir de G Suite for Education (Railean, 2012; Google, 2019) y adaptada a las necesidades docentes de un centro educativo de la ciudad de Valencia con 
1.300 alumnos y 80 profesores, con la finalidad de que el acondicionamiento a dicha plataforma por parte de los educadores sea lo más breve posible.

Cabe hacer notar que G Suite for Education es un conjunto de herramientas 2.0 que Google pone a disposición de los centros educativos de forma gratuita, para que puedan trabajar con un correo corporativo institucional y para crear un entorno de trabajo colaborativo dentro del propio centro (Silva, Veloso, Bento y Hernández, 2019).

Así, podemos establecer como objetivo la implementación de una plataforma de gestión educativa que se ajuste a los procesos de los docentes de un centro educativo mediante una aplicación, a ser posible gratuita y de fácil manejo para los docentes.

\section{MÉTOdO Y DISEÑO}

Las fases seguidas para la construcción de esta plataforma de gestión educativa propia, basada en G Suite for Education, fueron las que normalmente se utilizan en la mayoría de proyectos de desarrollo de software (Jacobson, Booch y Rumbaugh, 2000):

- Fase de análisis: La tarea principal de esta fase es la captura de requisitos. Estos deben delimitar exactamente cómo ha de funcionar la aplicación. En esta fase, se recopilaron cuáles eran las necesidades reales que tenían los distintos profesores. Para ello, las jefaturas de estudios de las etapas de Primaria y Secundaria-Bachiller-Ciclos Formativos comunicaron lo que esperaban que tuviera una herramienta de gestión académica para las necesidades concretas que presentaban el total de los 80 profesores de la institución. Otra fuente importantísima para recopilar información se basó en la propia experiencia como docentes de los autores. Así, se decidió que la plataforma debía estar formada por las siguientes herramientas:

1. Cuaderno del profesor, que permitiría al educador anotar todos sus procesos diarios (notas de exámenes, trabajo de clase, comportamiento del alumno, revisión del cuaderno, prueba inicial, etc.). 
2. Cuaderno digital a modo de dietario, en el que poder reflejar las tareas realizadas cada día, en una clase y materia concreta.

3. Listado de la clase para poder anotar las faltas, retrasos y faltas justificadas por los alumnos, en función de la hora docente del día, permitiendo obtener el porcentaje de faltas no justificadas por trimestre de cada uno de los alumnos.

4. Los tutores necesitaban algún documento en el que reflejar los datos de sus tutorizados, además de las reuniones realizadas con los padres de estos.

5. Acceso a una carpeta compartida, con todos los profesores, en la que el centro pudiera dejar información relevante común, como las orlas de cada una de las clases, documentos de calidad docente, manuales de la plataforma educativa, listados comunes y editables de las notas de los alumnos, etc.

6. Uso de calendarios comunes por etapas para que, de una forma eficaz, todos los estamentos supieran los actos y fechas más relevantes de la organización.

- Fase de diseño: En esta fase se buscaron las herramientas que Google, a través de G Suite for Education, aportaba y cómo ajustarlas en función del análisis previo realizado. Se llegó a la conclusión de que las herramientas a utilizar debían ser las siguientes:

1. Mediante las hojas de cálculo de Google se implementarían los siguientes elementos: cuaderno del profesor, dietario, listado de clase y documento de tutoría.

2. En Drive se crearía una carpeta compartida para que pudieran acceder todos los profesores, y visualizar y editar documentación relevante.

3. Se utilizarían los calendarios facilitados por Google, creando distintos grupos: grupo de Primaria, grupo de Secundaria, Bachiller, Ciclos y grupo de Colegio (con información general). De esta forma, un profesor pertenecería al grupo de Colegio y a Primaria, Secundaria, Bachiller o Ciclos, según la etapa en la que impartiera docencia. 
4. Por último, había que dotar a todas estas herramientas de una interfaz de fácil acceso. Para ello, se usó la herramienta Google Sites, que permite implementar webs visibles, o no, desde el exterior de la organización. En este caso, no sería visible, y cada profesor tendría su propia web, que le permitiría acceder a todas las herramientas anteriormente citadas de una manera fácil y visual.

- Fase de implementación: Se procedió a la realización de cada una de las herramientas:

1. Cuaderno del profesor: Se obtuvo un modelo de cuaderno del profesor Calvillo (2012) implementado originalmente para la aplicación ofimática de cálculo Excel. Este modelo se tuvo que adaptar para hojas de cálculo de Google, así como para los requerimientos de los profesores del colegio. Se crearon dos documentos base, uno para Primaria y otra para Secundaria-Bachiller-Ciclos Formativos, ya que los criterios de cada etapa no eran exactamente los mismos. En la siguiente figura se muestra el cuaderno de profesor de SecundariaBachiller-Ciclos: 






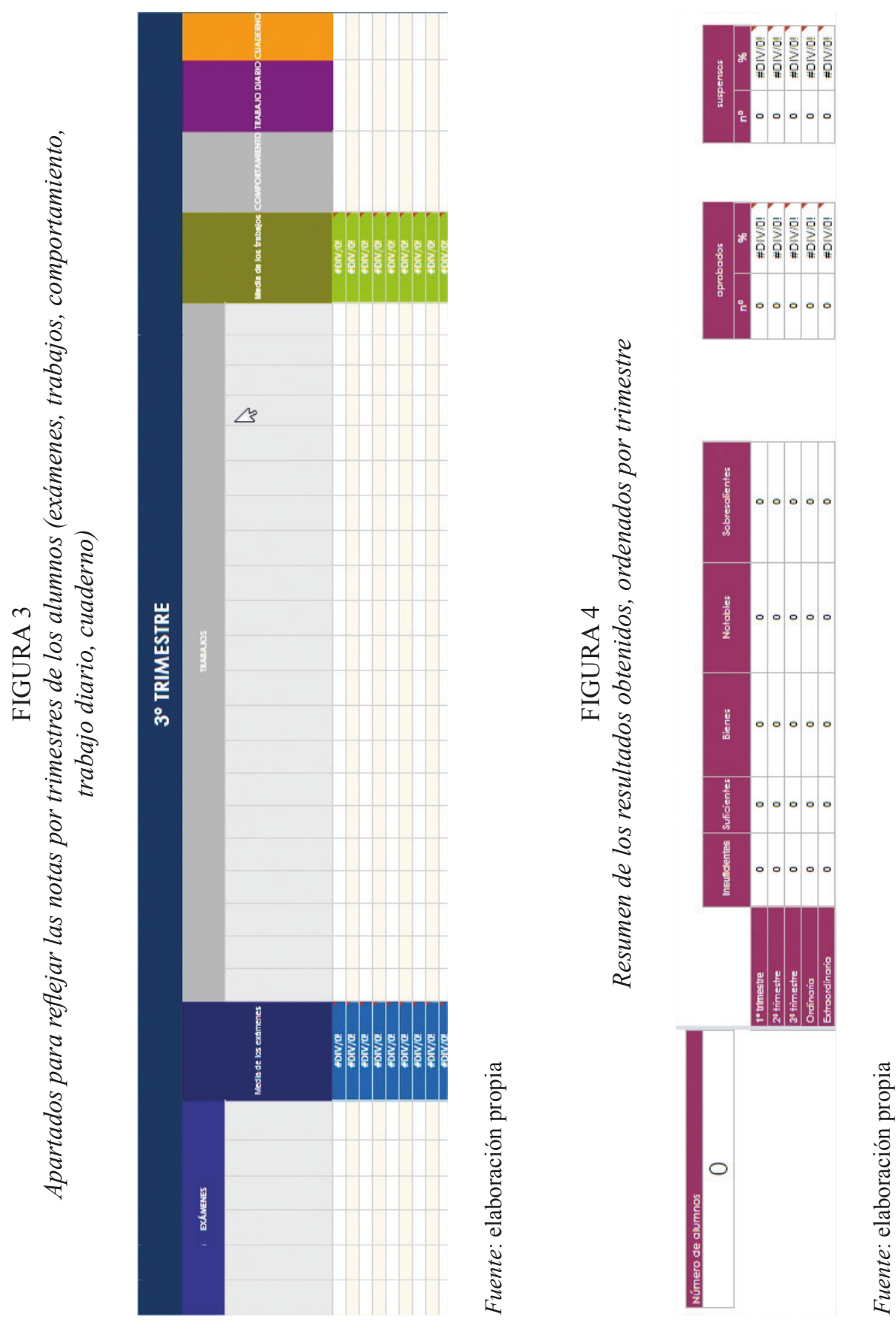
En este cuaderno del profesor, se pueden fijar las ponderaciones por evaluación a cada uno de los procesos docentes: trabajo de clase, comportamiento, prueba inicial (si la hubiera), cuaderno del alumno, exámenes y trabajos. La aplicación devuelve la nota calculada, según la ponderación, para cada trimestre y el docente redondeará la nota a un número entero (ya que la administración obliga a poner notas con números enteros) en la columna establecida para ello. Al final de la hoja, a modo de resumen, saldrán de forma automática la cantidad de aprobados, suspendidos, bienes, notables y sobresalientes, por cada una de las evaluaciones.

Obviamente, cada una de estas hojas está preparada para albergar todos los datos que el docente precisa para cada uno de los trimestres. De igual forma, el docente puede duplicar la plantilla base tantas veces como el número de materias que imparte, para tener una hoja por cada grupo.

2. Dietario: Se llevó a cabo mediante hojas de cálculo de Google. El profesor puede anotar lo que hace cada día en clase, con un determinado grupo: ejercicios, fechas de examen, la página del libro por la que se quedó, etc.

\section{FIGURA 5}

Dietario en el que los profesores pueden anotar lo sucedido en el día, en una materia en concreto

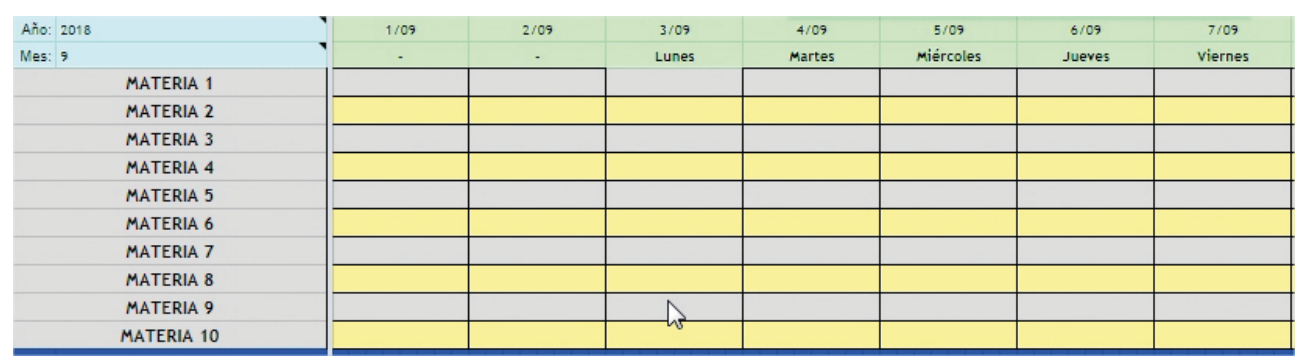

Fuente: elaboración propia 
3. Tutorías: También desarrolladas mediante hojas de cálculo de Google, partiendo de la plantilla de De Haro (2010). Al abrir dicha hoja se muestra el nombre y apellidos de todos los alumnos pertenecientes a un tutor en concreto, junto con las reuniones hechas y las fechas de las próximas entrevistas.

FIGURA 6

Documento de tutorías (vista principal)

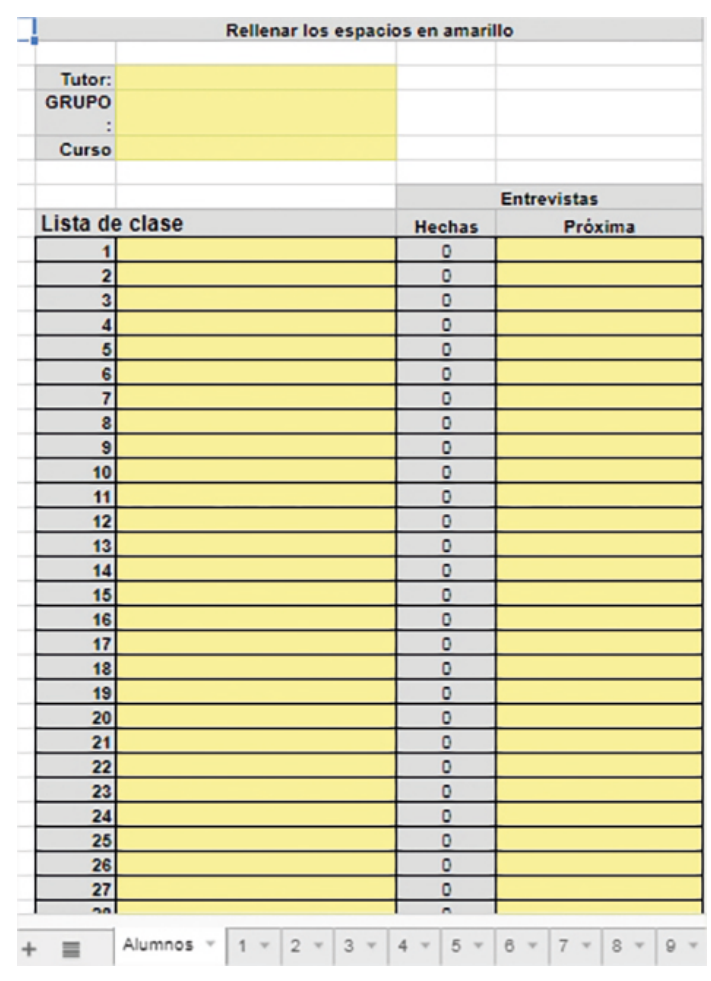

Fuente: elaboración propia

Tras la vista general de todos los alumnos, se puede acceder a la información personal de cada uno de ellos, seleccionando la hoja que se corresponde con su posición en el listado de clase, es decir, se podrá acceder a la información del alumno número 5 si se selecciona la hoja número 5 . 


\section{FIGURA 7}

Documentos de tutorías (ficha de cada alumno)

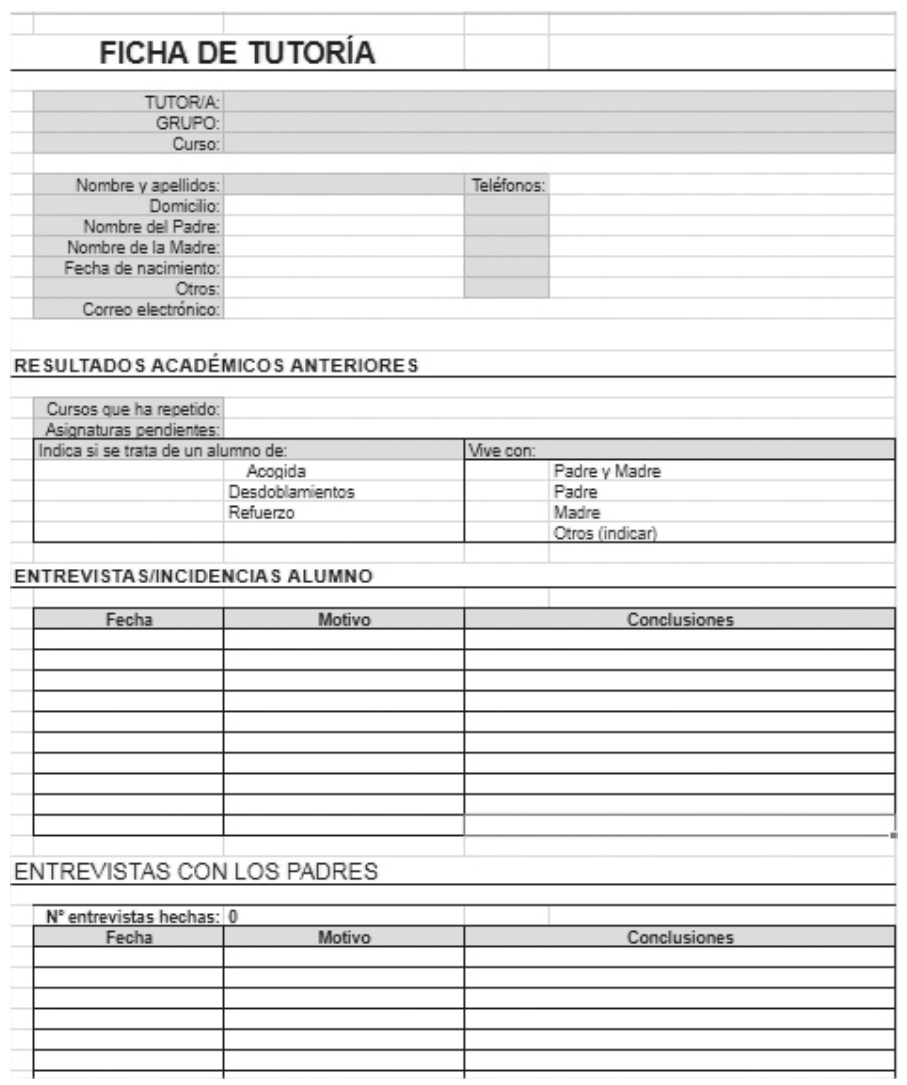

Fuente: elaboración propia

Como se puede apreciar, además de los datos personales de cada alumno (nombre del padre, madre, etc.), se pueden anotar los acuerdos alcanzados con los alumnos, al igual que los alcanzados con sus padres.

Cabe recalcar que conforme se van poniendo los emails de los padres de los alumnos, se va creando automáticamente una lista de distribución, ya que se van concatenando sus direcciones, separándolas con el símbolo ",". 
4. Lista de clase: Es sin duda el elemento más complejo, ya que un alumno, además de pertenecer a la lista de su clase, también puede pertenecer a una materia optativa, formada por alumnos del resto de grupos de ese nivel educativo. Esta situación exige que para obtener el total de faltas de un discente se debe acceder al total de listas de las asignaturas que conforman el nivel educativo y realizar la suma de faltas del individuo en el total de listas en las que aparece. Esta situación complica de forma extraordinaria la programación de la hoja de cálculo. Para ello, se tuvo que utilizar la función Vlookup (en Drive) que equivale a BuscarV (en Excel), junto con la función Importrange, para acceder a los distintos listados; e ir buscando el nombre de cada uno de los alumnos para poder sumar sus faltas diarias en todas las materias a las que accede cada día.

FIGURA 8

Recuento de faltas por trimestres

\begin{tabular}{|c|c|c|c|c|c|c|c|c|c|c|c|c|c|c|c|c|c|}
\hline \multicolumn{18}{|c|}{ Por favor, lee la hoja de AYUDA, que está al final, antes de empezar } \\
\hline & Trimestre 1 & \multicolumn{4}{|c|}{ T1 Septiembre } & \multicolumn{4}{|c|}{ T1 Octubre } & \multicolumn{4}{|c|}{ T1 Noviembre } & \multicolumn{4}{|c|}{ Total } \\
\hline & Mes: & \multicolumn{4}{|c|}{$9 / 2018$} & \multicolumn{4}{|c|}{$10 / 2018$} & \multicolumn{4}{|c|}{$11 / 2018$} & \multicolumn{4}{|c|}{ Trimestre 1} \\
\hline & Horas lectivas: & & & & & & & & & & & & & & & 0 & \\
\hline & Lista de alumnos & $\mathrm{F}$ & $\mathrm{J}$ & $R$ & $\% \mathrm{~F}+\mathrm{J}$ & $\mathrm{F}$ & $\mathrm{J}$ & $R$ & $\% \mathrm{~F}+\mathrm{J}$ & $\mathrm{F}$ & $\mathrm{J}$ & R & $\% \mathrm{~F}+\mathrm{J}$ & $\mathrm{F}$ & $\mathrm{J}$ & $\mathbf{R}$ & $\% \mathrm{~F}+\mathrm{J}$ \\
\hline 1 & & & & & & & & & & & & & & & & & \\
\hline 2 & & & & & & & & & & & & & & & & & \\
\hline 3 & & & & & & & & & & & & & & & & & \\
\hline 4 & & & & & & & & & & & & & & & & & \\
\hline 5 & & & & & & & & & & & & & & & & & \\
\hline 6 & & & & & & & & & & & & & & & & & \\
\hline
\end{tabular}

Fuente: elaboración propia

En las hojas denominadas RT1, RT2 y RT3, tal y como se muestra en la figura 8, aparece el recuento de faltas por meses y trimestres de cada uno de los alumnos. Si se debe poner a un alumno una falta, por ejemplo, en el mes de septiembre, únicamente hay que acceder a dicho mes y seleccionar la hora en la que ha faltado; se pondrá $\mathrm{F}$, J o R, según tenga una falta injustificada, justificada o retraso, respectivamente: 
FIGURA 9

Hoja para poner faltas a cada alumno por día y hora de clase



Fuente: elaboración propia

Para poder editar cada listado, los profesores acceden a una carpeta compartida en la que se encuentran todos los listados por cursos. El docente solo tiene que seleccionar el grupo en el que se encuentra en esa hora docente y pasar lista.

5. Carpeta compartida común: Se da acceso a una carpeta compartida, con derechos de edición, para que los profesores puedan entrar y trabajar con las orlas de clases, manuales de esta plataforma, procedimientos de calidad y documentos de evaluación.

6. Interfaz accesible: Cada profesor tendrá su propia web personalizada que le sirve de entrada a su plataforma, creada mediante Google Sites. Además, al abrir su web aparecerá el calendario de su grupo concreto y general del colegio. El docente solo deberá ir pinchando en los iconos de su web para acceder a los elementos descritos anteriormente.

- Fase de pruebas: Se realizaron todas las pruebas en los meses de mayo y junio de 2016. En julio de ese mismo año, se colgaron todas las herramientas en la hoja de plantillas de la plataforma de gestión y se procedió a dar la formación pertinente a los profesores.

- Fase de documentación: Se realizó toda la documentación necesaria durante los meses de pruebas. Esta se encuentra en la carpeta compartida a la que pueden acceder todos los profesores. 




EDETANIA 56 [Diciembre 2019], 185-202, ISSN: 0214-8560 


\section{Desarrollo del trabajo}

Los cimientos de la plataforma se comenzaron a construir desde noviembre de 2015, siguiendo todas las fases que se han descrito en la metodología. Lo esencial era que dicha plataforma se ajustara a los docentes y a los procesos que estos empleaban en sus clases, en las tutorías, en las comunicaciones, etc.

El otro gran pilar era utilizar una herramienta con la que los docentes estuvieran familiarizados y esta, sin duda, en el centro que nos atañe, era $G$ Suite for Education. Dicha aplicación se comenzó a utilizar en 2011, luego los profesores, en 2016, ya estaban muy familiarizados con la plataforma. Además, que esta herramienta sea gratuita para los centros educativos también suponía un acicate por el ahorro económico que iba a suponer.

En septiembre de 2016 todos los profesores de Primaria, Secundaria, Bachiller y Ciclos Formativos comenzaron a usar la plataforma. Para ello se realizó una sesión de presentación e implementación de aproximadamente tres horas por cada una de las etapas educativas. El objetivo era que al finalizar dicha sesión cada profesor tuviera implementada su propia plataforma de gestión de aula.

En primer lugar, descargaron la plantilla (figura 10) que les serviría de interfaz de acceso a su propia plataforma. Lo único que tuvieron que hacer fue personalizar dicha web con su nombre y configurarla para evitar que estuviera compartida con el resto de docentes de la institución. De esta forma, la web sería privada, ofreciéndoles el acceso a los distintos documentos mostrados en el capítulo de metodología.

Posteriormente, cada uno creó una carpeta en su Drive para introducir el cuaderno de profesor, el documento de tutorías y el dietario. Todos estos documentos eran accesibles a través de las plantillas creadas en Drive, es decir, que el profesor lo único que debía hacer era descargarlas desde su cuenta. Posteriormente, tuvieron que activar la edición de su Google Sites y vincular cada uno de los documentos citados con cada icono de esta. De esta forma, podrían acceder a los distintos elementos con un clic partiendo de su web personalizada.

El acceso a la carpeta compartida común y a los listados de faltas ya venía establecido en la propia plantilla de Google Sites, al igual que su calendario. En definitiva, la implantación y el manejo de la plataforma por los 80 docen- 
tes supusieron un total de seis horas de formación. En esta, aprendieron el manejo de la aplicación y la pudieron personalizar a su gusto.

\section{Conclusiones}

El objetivo de crear una plataforma que se ajustara a los procesos de los docentes a partir de una herramienta potente y que no supusiera una gran curva de aprendizaje estaba cumplido.

Anteriormente, se utilizaba una plataforma comercial de las que se comentaron en la introducción y solamente su implementación (introducir horarios, grupos, etc.) supuso una gran dedicación de tiempo: alrededor de 50 horas a un total de 5 personas. A esto hay que añadir la formación general al total de docentes para adaptarse a la plataforma, lo que supone que los procesos se adapten a una herramienta, en vez de que la herramienta sea la que se adecue a la forma de trabajar de los profesores.

Tras la utilización de la plataforma de gestión propia durante tres cursos docentes, se puede concluir que se han agilizado enormemente todos los procesos de comunicación a través del correo electrónico corporativo y de los calendarios compartidos, con la posibilidad de sincronización en el smartphone.

Hay que destacar la capacidad de adaptación de la herramienta a cada uno de los profesores; es decir, con su uso sucesivo, cada docente ha ido adaptando las plantillas de inicio a sus procesos educativos.

Las tutorías han mejorado sustancialmente porque los profesores pueden compartir (con la opción de ver) su cuaderno del profesor, luego el tutor dispone de todas las notas de un alumno de previamente a la reunión con los padres.

El conteo de faltas de los alumnos ha mejorado exponencialmente, pasando de la anotación en una plantilla en papel a obtener el número de faltas, incluidos porcentajes de asistencia de forma automática.

Con el acceso a una carpeta compartida por toda la comunidad educativa se han facilitado todos los procesos. Por ejemplo, en esta carpeta se ponen las orlas de cada una de las clases para que los profesores puedan hacer sus listados y poner cara a los alumnos. Se comparte una plantilla para que todos los docentes puedan poner sus notas, de forma previa, a las sesiones de 
evaluación. Se facilitan múltiples plantillas para proponer actividades complementarias, aceptación de las salidas por parte de las familias, documentos de calidad educativa, manuales y videotutoriales de funcionamiento de la plataforma de gestión, etc.

En este último curso 2018-2019, se ha fomentado el uso de la aplicación Google Classroom para fomentar la interactividad con el alumnado. Esto ha supuesto añadir una funcionalidad educativa a la plataforma que inicialmente estaba pensada para la gestión. Para que los alumnos pudieran tener la máxima funcionalidad en Classroom, se les ha creado una cuenta de email institucional, con la peculiaridad de que dicha cuenta únicamente sea válida para comunicarse con personas con cuentas del mismo dominio. Es decir, este correo únicamente les permite establecer contacto con sus compañeros y con sus profesores. Así, además de proporcionarles toda la funcionalidad con la herramienta Google Classroom, también les estamos ofreciendo un canal de comunicación y la posibilidad de almacenamiento en la nube (Drive). Cabe recalcar que, dado que es una cuenta G Suite for Education, el almacenamiento del que disponen es infinito y sin publicidad (Google, 2019).

Actualmente, ningún docente utiliza un documento de gestión en formato papel, e incluso han manifestado que el uso de la plataforma les ha supuesto un aliciente y una novedad en su actividad docente diaria.

También se debe destacar el ahorro económico que ha supuesto para el colegio esta plataforma de gestión propia, ya que todas las herramientas son gratuitas. Sin embargo, en su implementación se ha invertido un enorme número de horas, sobre todo en la herramienta para poder pasar lista.

Para terminar, hay que destacar que la plataforma sigue viva gracias a las sugerencias que van realizando los docentes y que poco a poco se van incorporando.

\section{BiBLIOGRAFÍA}

AdDitio App (2019). Additio App for Schools. Recuperado de: https://www. additioapp.com/es/plataforma-centros/.

AleXia (2019). Qué es Alexia. Recuperado de: http://www.alexiaeducaria. $\mathrm{com} /$ suite-educativa/. 
Canós, L., Canós, M. J. y Liern, V. (2009). El uso de las nuevas tecnologías aplicadas a la Educación Superior. XVII Jornadas ASEPUMA - V Encuentro Internacional.

Calvillo, A. (2012). Cuaderno del profesor en Excel muy fácil más manual. Recuperado de: http://www.musikawa.es/cuaderno-del-profesor-en-excelmuy-facil-manual-musikawa/.

Clickedu (2019). ¿Qué es Clickedu? Recuperado de: https://clickartedu.com/ inicio-plataforma-colegios.html.

De Haro, J. J. (2010). Algunas plantillas de Google Docs para usar en clase. Recuperado de: https://jjdeharo.blogspot.com/.

EdUCACIÓN 3.0. (2018). 35 plataformas para la gestión de centros educativos. Recuperado de: http://www.educaciontrespuntocero.com/novedades2/ software2/plataformas-gestion-escolar/12663.html.

Google (2019). G Suite for Education. Recuperado de: https://edu.google. com/int1/es-419/products/gsuite-for-education/?modal_active=none.

Google (2019). Preguntas frecuentes sobre G Suite para Centros Educativos. Recuperado de: https://support.google.com/a/answer/139019?hl=es.

Jacobson, I., Booch, G. y Rumbaugh, J. (2000). El proceso unificado de desarrollo de software. Nueva York: Pearson Educación.

MuÑoz-Iglesias, M. y SANTini, S. (2015). Gestión académica y de comunicación directa entre centros educativos y familias. Recuperado de: https:// repositorio.uam.es/bitstream/handle/10486/668818/Mu\%C3\%B1oz Iglesias_Marcos_tfg.pdf?sequence $=1$.

RAILEAN, E. (2012). Google Apps for Education - a powerful solution for global scientific classrooms with learner-centred environment. International Journal of Computer Science Research and Application 2, 19-27.

Silva, S., Veloso, M., Bento, V. y Hernández, L. (2019). La teoría de las generaciones entre los profesores universitarios: La percepción de calidades de Google Apps for Education. Revista Cientifica Hermes 23, 98-121.

SM (2019). Gestión de centros. Recuperado de: http://www.educamos.com/ conoce.html. 